\title{
Reaction of Lithioamines with Alkyl Halides: A Convenient Direct Synthesis of $\mathrm{N}$-Alkylaminopyridines
}

\author{
Okram Mukherjee Singh, ${ }^{*}$ S. Joychandra Singh, Su Nam Kim, ${ }^{\dagger}$ and Sang-Gyeong Lee ${ }^{*, *}$ \\ Department of Chemisty; Manipur Lniversity, Canchipur-795003, Manipur, India \\ "Department of Chemistry \& Research Institute of Life Science, Gyeongsang National liniversity, Jinh, \\ GyeongNam 660-701, Korea. "E-mail: leesangíagsmack kr \\ Received Octoher 10.2006
}

Key Words : $N$-Alkylation. Lithioaminoanions, $N$-Alkylated heteroaromatic amines

$N$-Alkylation of aromatic and heteroaromatic amines constitutes an important synthetic strategy for new C-N bond formation. Aromatic and heteroaromatic amines are less nucleophilic when compared to aliphatic amines and alkylation of these amines requires activated halides, alcohols, aldehydes and ketones or expensive metal catalysts to undergo the $\mathrm{C}-\mathrm{N}$ bond formation process. ${ }^{1-7}$ Generally when alkyl halides are used as alkylating agents, the reactions proceed slowly, and several polyalkylation/halogenated byproducts are observed. ${ }^{8}$

Cross coupling reactions of aryl halides with aromatic and heteroaromatic amines using palladium catalyzed C-N coupling protocol emerges as a new for $N$-alkylation and $N$ arylation. ${ }^{74} \mathrm{ln}$ another recent paper lowary of $a^{1 l^{10}}$ demonstrated a three-step process for $N$-monoalkylation of 2aminopyridines, which involves initially protection of amino group as $N$-Boc.

However many of the reported methods used complicated multistep reactions, under drastic reaction conditions, or via coupling reactions using expensive metal catalysts. Many of these methods leads to mixtures of secondary and tertiary amines that are difficult to separate.' To prevent overalkylation, troublesome and expensive multistep methods have been derived such as partial protection of primary amines" and reduction of mono $N$-substituted amides..$^{12}$ Although there were known good methods ${ }^{13-15}$ for synthesis of $N$ alkylation of aromatic and heteroaromatic amines, still improved selective methods continue to be sought.

According to reported paper, ${ }^{13-15}$ we investigated the $N$ monoalkylation of aminopyridine at $-78^{\circ} \mathrm{C}$ to $-40^{\circ} \mathrm{C}$. However, during the experiment, we recognized that the generated lithioaminoanion from the reaction of aminopyridine with $n$-butyllithium is stable for $1 \mathrm{~h}$ at $0^{\circ} \mathrm{C}$ to $25^{\circ} \mathrm{C}$.

Herein we report a general method of $N$-alkylation of aromatic and heteroaromatic amines 1 by treating the lithioaminoanions 2 generated at $0^{\circ} \mathrm{C}$ to $25^{\circ} \mathrm{C}$ in situ in presence of $n$-butyllithium with various alkyl halides (Scheme 1). The reactions are clean, mild, and high yields of the alkylated amines 3 are produced in very short reaction time.

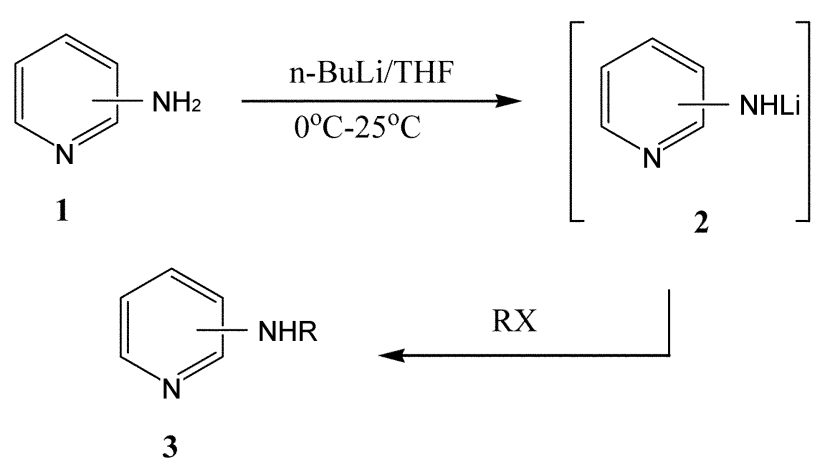

Scheme 1<smiles>Nc1ccccn1</smiles>

4

$$
\underset{\text { 1) } \mathrm{n}-\mathrm{BuLi} / 0^{\circ} \mathrm{C}-25^{\circ} \mathrm{C}}{\mathrm{RX}, \text { ambient temp. }}
$$

The reaction of 2-aminopyridine $\mathbf{4}$ with primary alkyl halides yielding $N$-monoalkylated pyridines in excellent yields was first investigated at room temperature (Scheme 2).

The generation of lithioamino anion was indicated by the appearance of a reddish-brown colour after adding butyllithium, which was initially a colourless solution in THF. The same process was successfully extended to 3-aminopyridine and 4-aminopyridines using more or less similar alkyl halides. The corresponding 3-alkylaminopyridines and 4-alkylaminopyridines were obtained in good to excellent yields at $0^{\circ} \mathrm{C}$ to $25^{\circ} \mathrm{C}$. The results are listed in Table 1 .

We tried to get $N$-monoalkylated aminopyrodines by reaction with lithioamino anion and alkyl chlorides or alkyl bromides. But the reaction time was retarded to compare with alkyl iodides and some amount of $N$-dialkylated aminopyridines were obtained as byproduct with desired product. 
Table 1. Synthesis of $\mathrm{V}$-alkylaminopyridines from aminopyridines and alkyl halides

\begin{tabular}{cccc}
\hline Entry & Amine & Halide (RX) & $\begin{array}{c}\text { Product } \\
\text { (yield, \%) }\end{array}$ \\
\hline 1 & 2-Aminopyridine & Methyl iodide & $\mathbf{5 a}(85)$ \\
2 & 2-Aminopyridine & Ethyl iodide & $\mathbf{5 b}(75)$ \\
3 & 2-Aminopyridine & $n$-Propyl iodide & $\mathbf{5 c}(75)$ \\
4 & 2-Aminopyridine & $n$-Butyl bromide & $\mathbf{5 d}(80)$ \\
5 & 2-Aminopyridine & Benzyl bromide & $\mathbf{5 e}(85)$ \\
6 & 3-Aminopyridine & Methyl iodide & $\mathbf{5 f}(73)$ \\
7 & 3-Aminopyridine & Ethyl iodide & $\mathbf{5 g}(75)$ \\
8 & 4-Aminopyridine & Methyl iodide & $\mathbf{5 h}(74)$ \\
9 & 4-Aminopyridine & Ethyl iodide & $\mathbf{5 i}(80)$ \\
\hline
\end{tabular}

In summary, we have established a one pot direct $N$ alkylation process for several heteroaromatic amines using $n$-butyllithium at $0{ }^{\circ} \mathrm{C}$ to $25^{\circ} \mathrm{C}$. It is worth mentioning that reaction conditions are mild. clean and within a shorter reaction time the yields of the $N$-alkylated heteroaromatic amines were excellent.

\section{Experimental Section}

Typical synthetic procedure for the $N$-alkylation of heteroaromatic amines: To a stirred solution of 2-aminopyrridine $(0.05 \mathrm{~mol})$ in dry THF $(40 \mathrm{~mL})$, n-butyllithium (0.05 mole) was added under $\mathrm{N}_{2}$ atmosphere at $0^{\circ} \mathrm{C}$ and continued stirring for $30 \mathrm{~min}$. The temperature of the reaction mixture increased to room temperature and alkyl halide was added slowly (in case solid substrates THF solution was used). The reaction mixture was stirred at the ambient temperature for $1 \mathrm{hr}$. The reaction mixture was poured to water. $\mathrm{NH}_{4} \mathrm{Cl}$ and extracted with chloroform $(150 \mathrm{~mL})$. The chloroform extract was dried $\left(\mathrm{Na}_{2} \mathrm{SO}_{4}\right)$ and evaporated on a rotary evaporator to yield a residue which was either distilled or crystallized to afford the corresponding 2-alkylamino pyridines in 75-85\% overall yields (Table 1). All of the obtained compounds 5a-5i were synthesized similarly and the spectroscopic data are as follows.

2-(Methylamino)pyridine (5a). Colorless oil. IR (neat): 3268,3042 . 2941, 1603. 1520,1411. 1330, 1289. $1154 \mathrm{~cm}^{-1}$. ${ }^{1} \mathrm{H}$ NMR (300 MHz, $\mathrm{CDCl}_{3}$ ) 8.09 (ddd, I H, $J=5.0 .2 .0 .0 .8$ Hz). 7.42 (ddd. $1 \mathrm{H}, J=8.4,7.1 .2 .0 \mathrm{~Hz}$ ), 6.56 (ddd, $1 \mathrm{H} . J=$ 7.1. $5.0 .0 .8 \mathrm{~Hz}), 6.37$ (ddd $1 \mathrm{H}, J=8.4 .0 .8 .0 .8 \mathrm{~Hz}), 4.64$ (br. s. $1 \mathrm{H}) .2 .91$ (d. $3 \mathrm{H} . J=5.1 \mathrm{~Hz}) .{ }^{13} \mathrm{C}$ NMR $(75.5 \mathrm{MHz}$. $\left.\mathrm{CDCl}_{3}\right)$ 29.1, 106.2. 112.7, 137.4, 148.2. 159.7. Anal. Calcd for $\mathrm{C}_{6} \mathrm{H}_{8} \mathrm{~N}_{2}:$ C, 66.64: H, 7.46. Found: C. 66.75; H. 7.12.

2-(Ethylamino)pyridine (5b). ${ }^{1} \mathrm{H}$ NMR (400 MHz): $\delta$ $1.20($ t. $J=7.2 \mathrm{~Hz} .3 \mathrm{H}), 3.20(\mathrm{q}, J=6.8 \mathrm{~Hz} .2 \mathrm{H}), 5.32$ (br. s. $1 \mathrm{H}), 6.33(\mathrm{~d}, J=8.4 \mathrm{~Hz} . \mathrm{lH}) .6 .50(\mathrm{t} . J=8.8 \mathrm{~Hz}, 1 \mathrm{H}) .7 .38$ $(\mathrm{t} J=1.6 \mathrm{~Hz}, 1 \mathrm{H}) .7 .93(\mathrm{~d}, J=5.2 \mathrm{~Hz}, 1 \mathrm{H}),{ }^{13} \mathrm{C}$ NMR $(100$ $\mathrm{MHz}): \delta 14.7,36.8,106.1$. 113.0, 137.2. 148.0, 159.1: Mass $m / z=122\left(\mathrm{M}^{+}\right)$.

2-(Propylamino)pyridine (5c). ${ }^{\mathrm{H}} \mathrm{H}$ NMR (400 MHz): $\delta$ 0.995 (t. $J=7.2 \mathrm{~Hz} .3 \mathrm{H}$ ). $1.64(\mathrm{~m} .2 \mathrm{H}), 3.21$ (q. $J=6.0 \mathrm{~Hz}$. $2 \mathrm{H}$ ). 4.60 (br. s, $1 \mathrm{H}), 6.37$ (d. $J=8.4 \mathrm{~Hz} .1 \mathrm{H}) .6 .54(\mathrm{~m}, \mathrm{lH})$. $7.41(\mathrm{~m} .1 \mathrm{H}) .8 .07(\mathrm{~d} . J=4.8 \mathrm{~Hz} .1 \mathrm{H}):{ }^{13} \mathrm{C}$ NMR $(100 \mathrm{MHz})$ $\delta$ 11.4, 22.6. 44.0. 106.1. 112.3. 137.1. 148.0. 159.2: Mass $m / z=136\left(\mathrm{M}^{-}\right)$

2-(Butylamino)pyridine (5d). ${ }^{\mathrm{H}} \mathrm{H}$ NMR (400 MHz): $\delta$ $0.953(\mathrm{t}, J=7.6 \mathrm{~Hz} .3 \mathrm{H}) .1 .42(\mathrm{~m}, 2 \mathrm{H}) .1 .6 \mathrm{l}(\mathrm{m}, 2 \mathrm{H}), 3.24$ (q. $J=6.4 \mathrm{~Hz}, 2 \mathrm{H}), 4.55$ (br. s. $1 \mathrm{H}), 6.36(\mathrm{~d} J=8.0 \mathrm{~Hz} .1 \mathrm{H})$. $6.54(\mathrm{~m} .1 \mathrm{H}), 7.4$ (t. $J=7.2 \mathrm{~Hz}, 1 \mathrm{H}), 8.07$ (d. $J=4.8 \mathrm{~Hz}$. $1 \mathrm{H}):{ }^{13} \mathrm{C}$ NMR (100 MHz): $\delta 13.8 .20 .2,31.6 .42 .0 .106 .1$, 113.0. 137.2. 148.3, 159.1: Mass $m / z=150\left(\mathrm{M}^{+}\right)$.

2-(Benzylamino)pyridine (5e). Colourless solid. mp 94$96^{\circ} \mathrm{C}$ IR: $3225,3024,2868,1600,1528,1442.1334,1152$. $1079 \mathrm{~cm}^{-1}$ : ${ }^{1} \mathrm{H}$ NMR $\left(500 \mathrm{MHz} . \mathrm{CDCl}_{3}\right): \delta 8.09$ (ddd, lH, $J$ $=4.9 .1 .9 .0 .9 \mathrm{~Hz}), 7.38$ (ddd. $1 \mathrm{H}, J=8.4,7.0,1.9 \mathrm{~Hz}) .7 .35-$ $7.31(\mathrm{~m} .4 \mathrm{H}), 7.28-7.25$ (n. $1 \mathrm{H}), 6.57$ (ddd. $1 \mathrm{H} . J=7.0 .4 .9$. $0.9 \mathrm{~Hz}$ ). 6.35 (ddd. $1 \mathrm{H}, J=8.4,0.9,0.9 \mathrm{~Hz}$ ) 4.95 (br. s. $1 \mathrm{H}$ ). 4.49 (d. $2 \mathrm{H}, J=5.6 \mathrm{~Hz}$ ): ${ }^{13} \mathrm{C}$ NMR (125 MHz, $\left.\mathrm{CDCl}_{3}\right): \delta$ 46.3. 106.8, 113.1, 127.2. 127.4. 128.6, 137.4. 139.2. 148.2, 158.7. Anal. Calcd for $\mathrm{C}_{12} \mathrm{H}_{12} \mathrm{~N}_{2}: \mathrm{C}, 78.23: \mathrm{H}, 6.56$. Found: C. 78.40: H. 6.68: HRMS (ESI) calcd for $\left(\mathrm{M}+\mathrm{H}^{-}\right.$) $\mathrm{C}_{12} \mathrm{H}_{12} \mathrm{~N}_{2}$ : 185.1073 : Found: 185.1062.

3-(Methylamino)pyridine (5f). ${ }^{~} \mathrm{H}$ NMR (400 MHz): $\delta$ 2.68 (s. $3 \mathrm{H}$ ). 6.64 (br. s, lH). 6.85 (d. $J=7.0 \mathrm{~Hz} . \mathrm{lH}), 7.13$ (dd. $J=7.0,7.5 \mathrm{~Hz}, 1 \mathrm{H}) .7 .93(\mathrm{~d}, J=7.5 \mathrm{~Hz}, 1 \mathrm{H}), 7.95$ (s. $1 \mathrm{H}):{ }^{13} \mathrm{C}$ NMR (100 MHz): $\delta 29.5,122.9 .124 .5 .146 .0$. 137.6. 138.8: Mass $m / z=108\left(\mathrm{M}^{+}\right)$.

3-(Ethylamino)pyridine (5g). ${ }^{1} \mathrm{H}$ NMR $(400 \mathrm{MHz}) ; \delta$ $1.24(\mathrm{t} . J=8.4 \mathrm{~Hz} .3 \mathrm{H}$ ), 3.15 (q. $J=7.2 \mathrm{~Hz} .2 \mathrm{H}$ ). 3.83 (br. s, 1H). $6.84(\mathrm{~d}, J=8.0 \mathrm{~Hz}, 1 \mathrm{H}) .7 .06(\mathrm{~m}, 1 \mathrm{H}) .7 .93(\mathrm{~d} J=4.8$ $\mathrm{Hz}, 1 \mathrm{H}), 8.01(\mathrm{~d} J=4.8 \mathrm{~Hz}, 1 \mathrm{H}):{ }^{13} \mathrm{C}$ NMR $(100 \mathrm{MHz}): \delta$ 14.5. 37.9, 118.1. 124.3. 136.6, 138.1, 144.2; Mass $m / z=$ $122\left(\mathrm{M}^{-}\right)$.

4-(Methylamino)pyridine (5h). ${ }^{1} \mathrm{H}$ NMR (400 MHz): $\delta$ 2.47 (s. 3H). 6.63 (br. s, lH), 6.64 (m, 2H), 8.43 (m. 2H); ${ }^{13}$ CNMR (100 MHz): $\delta 29.5$. 109.1. 109.4. 150.2, 150.4, 154.9: Mass $m / z=108\left(\mathrm{M}^{+}\right)$.

4-(Ethylamino)pyridine (5i). ${ }^{1} \mathrm{H}$ NMR $(400 \mathrm{MHz}): \delta$ 1.25 (t. $J=7.2 \mathrm{~Hz}, 3 \mathrm{H}), 3.17$ (q. $J=5.2 \mathrm{~Hz}, 2 \mathrm{H}$ ). 4.28 (br s. 1H). $6.44(\mathrm{~m} .2 \mathrm{H}) .8 .17(\mathrm{~m}, 2 \mathrm{H}):{ }^{13} \mathrm{C} \mathrm{NMR}(100 \mathrm{MHz}): \delta$ 14.3. 37.1. 107.0. 109.3. 150.1; Mass $m / z=122\left(\mathrm{M}^{+}\right)$.

Acknowledgements. OMS \& SIS thank CSIR. New Delhi for funancial assistance and CDRI. Luck now for some of the NMR recordings. Lee thanks to Gyeongsang National University for his sabbatical year program in 2005.

\section{References and Notes}

1. Burdon. T.: McLonghlim. V. C. R. Tetrahedron 1965. 2l. 1-4

2. Tanigawa. Y: Murahashi. S.: Moritani. I. Tetrohedron Lett. 1975. $471-472$

3. Murahashi. S.: Kondo. K.: Hakata. T. Tetrahedron Lett. 1982. 23. 229-232

4. Grigg. R: Mitchell, T. R. B: Suthivaiyakit. S: Tongpenyai, N.d. Chem. Soc. Chem. Commun 1981,611-612.

5. Arcelli. A.: Khai. B.-T.: Porzi. G. J. Organomet. Chent 1982. 235. $93-96$.

6. (a) Watanabe. Y.: Tsuiji. Y.: Ohsugi. Y. Tenohedron Lett. 1981. 22. 2267-2268. (b) Watanabe, Y.: Tsuji. Y: Ige, H.: Ohsugi. Y: Ohta. T. J. Org. Chem 1984. 19, 3359-3363. (c) Watanabe. Y.; Morisaki. 
Y: Kondo. T.: Mitsudo. T-a. J. Org Chem. 1996. 61, 4214-4218. (d) Abbenhuis. R. A. T. M.: Boersma. J.: Koten. G. v. J. Org. Chem. 1998.63.4282-4290.

7. (a) Antilla. J. C.: Baskin. J. M.: Barder. T. E.: Buchwald. S. L. J. Org. Chem. 2004, 69, 5578-5587. (b) Muci. A. R. Buchwald. S. L. Top. Cwr Chem 2002. 219.131-209. (c) Wolfe, J. P; Wagaw: S.: Marcoux J. F; Buchwald. S. L. Acc. Chem. Res. 1998, 31 , 805-818. (d) Hartwig. J. F. In Handtook of Organopalladium Chemistry for Organic Synthesis: Negishi. E.-i. Ed.: Wiley: New York. 2002: Vol. 1. pp 1051-1096. (e) Hartwig. J. F. Acc. Chem. Res. 1998. 31. 852-860.

8. Onaka, M: Umezono, A: Kawai, M: Izumi, Y. J. Chem. Soc. Chem. Conmm 1985. 17, 1202-1203

9. (a) Jiang. L.: Buchwald. S. L. In Metal-Catalized Cross-Coupling Reactions. 2nd ed.: de Meijere. A.. Diederich. F.. Eds.: John Wiley \& Sons: Weinheim. 2004. (b) Luker. T. J.: Beaton. H. G.: Whiting. M.: Mete, A.: Cheshire, D. R. Tetrahedron Lett. 2000. H1. 77317735. (c) Watanabe M.: Yamamoto, T.: Nishivama. M. Chem.
Conmun. 2000, 133-134. (d) Ogawa, K.: Radke, K. R.: Rothstein. S. D.: Rasmussen. S. C. J. Org. Chem. 2001. 66.9067-9070. (e) Hooper. M. W.: Utsunomiya. M.: Hartwig. I. F. J. Org. Chent. 2003. 68. 2861-2873. (f) Hooper. M. W.: Hatwig. T. F. Organometallics 2003, 22. 3394-3403.

10. Krein, D. M.: Lowary, T. L. J. Org. Chem 2002, 67. 49654967.

11. Selva. M.: Tundo. P.: Perosa. A. J. Org. Chem. 2001. 66. 677-680 and references therein.

12. (a) Larock. R. C. Comprehensine Organic Transformationts. 2nd ed, Wiley-VCH: New York, 1999; pp 869-874. (b) Mataloni. M. Petrini, M.: Profeta. R. Sylett. 2003, 1129-1132.

13. Depue. J. S.; Collum. D. B. J. Am. Chem. Soc. 1988. 110, 55245533.

14. Suga. K.: Watanabe S: Fujita. T.: Pant. T. P. Bull. Chent. Soc. Jpn. 1969. +2.3606-36008

15. Adima A.: Bied. C.: Moreau. J. J.; Man. M. W. C. Eur J. Org. Chem. 2004, 2582-2588. 